\title{
Purifying and Reversible Physical Processes
}

\author{
Matthias Kleinmann, Hermann Kampermann, Tim Meyer, and Dagmar Bruß \\ Institut für Theoretische Physik, Heinrich-Heine-Universität Düsseldorf, D-40225 Düsseldorf, Germany
}

August 8, 2018

\begin{abstract}
Starting from the observation that reversible processes cannot increase the purity of any input state, we study deterministic physical processes, which map a set of states to a set of pure states. Such a process must map any state to the same pure output, if purity is demanded for the input set of all states. But otherwise, when the input set is restricted, it is possible to find non-trivial purifying processes. For the most restricted case of only two input states, we completely characterize the output of any such map. We furthermore consider maps, which combine the property of purity and reversibility on a set of states, and we derive necessary and sufficient conditions on sets, which permit such processes.
\end{abstract}

\section{Introduction}

The notion of a pure quantum state plays a special role in quantum information theory. Many problems - such as separability or the existence of a particular quantum protocol - can easily be solved, if one restricts the problem to pure quantum states only. On the other hand mixed states endow quantum systems with many properties (such as bound entanglement), that cannot be found for systems described by pure states. In our contribution we investigate physical processes which transform a given set of mixed states to a set of pure states. If such a process exists, then it may e.g. be possible to infer from the properties of the pure output states some properties of the input states. Such a kind of conclusion is particular powerful, if the purifying map can be chosen to be reversible, since then one can consider the set of pure output states and the set of input states as physically equivalent.

We consider deterministic physical processes, i.e., processes which map any possible input quantum state with probability one to a corresponding output quantum state. The states of the input quantum system are represented by density operators $\rho_{\text {in }} \in \mathcal{B}_{+}\left(\mathcal{H}_{\text {in }}\right)$, i.e., positive semidefinite operators with trace one acting on the finite-dimensional complex vector space $\mathcal{H}_{\text {in }}$. Analogously $\rho_{\text {out }} \in \mathcal{B}_{+}\left(\mathcal{H}_{\text {out }}\right)$ shall represent the set of states of the output quantum system. Any deterministic physical process can be written as a completely positive and trace preserving linear (CPTP) map $\Lambda: \mathcal{B}\left(\mathcal{H}_{\text {in }}\right) \rightarrow$ $\mathcal{B}\left(\mathcal{H}_{\text {out }}\right)$, where $\mathcal{B}\left(\mathcal{H}_{\text {in,out }}\right)$ denotes the space of linear operators on $\mathcal{H}_{\text {in,out. }}$ In this language, the trace preserving condition reflects the fact that we restrict our considerations to deterministic processes. In Section 3 we will argue that this restriction is indeed necessary to have a proper definition of a purifying map.

A reversible process is a physical process, where the action of the process on any physical state can be undone by another physical process, i.e., a CPTP map $\Lambda$ is reversible if one can find an inverse map $\Lambda^{\prime}$ which is also CPTP and satisfies $\left(\Lambda^{\prime} \circ \Lambda\right)\left[\rho_{\text {in }}\right]=\rho_{\text {in }}$ for any density operator $\rho_{\text {in }}$. The most common example are unitary processes $E_{U}: \chi \mapsto U \chi U^{\dagger}$, where $U$ is a unitary transformation, $U U^{\dagger}=\mathbb{1}$. Here obviously the inverse map is given by $\left(E_{U}\right)^{\prime}=E_{U^{\dagger}}$. Another class of reversible processes that is important for our purposes is described by

$$
E_{\sigma}: \mathcal{B}\left(\mathcal{H}_{\text {in }}\right) \rightarrow \mathcal{B}\left(\mathcal{H}_{\text {in }} \otimes \mathcal{H}_{\text {aux }}\right): \chi \mapsto \chi \otimes \sigma
$$

where $\sigma \in \mathcal{B}_{+}\left(\mathcal{H}_{\text {aux }}\right)$ is some arbitrary density operator. The inverse map for this process is the partial trace over the auxiliary system, $\left(E_{\sigma}\right)^{\prime}=\operatorname{tr}_{\mathrm{aux}}$. A remarkable property of this inverse map is, that it does not depend on $\sigma$ and hence cannot be reversible. Note that neither the action of $E_{U}$ nor the one of $E_{\sigma}$ increases the purity $\operatorname{tr}\left(\rho^{2}\right)$ of any density operator $\rho$. Indeed a process, which is reversible on the set of all states cannot increase the purity of even a single state: Let us first consider reversible maps, for which the reverse map is the partial trace (e.g. $E_{\sigma}$ ). For such a reversible map $\Lambda$, the output of any pure state $\Pi_{|\phi\rangle} \equiv|\phi\rangle\langle\phi|$ must be $\Lambda\left[\Pi_{|\phi\rangle}\right]=\Pi_{|\phi\rangle} \otimes \sigma_{\phi}$ for some state $\sigma_{\phi}$. For any state $\rho_{\text {in }}$ we find with the spectral decomposition $\rho_{\text {in }}=\sum_{i} p_{i} \Pi_{\left|\lambda_{i}\right\rangle}$ that due to linearity, $\operatorname{tr}\left(\Lambda\left[\rho_{\text {in }}\right]^{2}\right)=\sum p_{i}^{2} \operatorname{tr}\left(\sigma_{\lambda_{i}}^{2}\right) \leq \operatorname{tr}\left(\rho_{\text {in }}^{2}\right)$, i.e., no state can become purer by the action of $\Lambda$. Now consider a general reversible map $\Lambda$. For the reverse process $\Lambda^{\prime}$, by virtue of Stinespring's dilation theorem [1,2] one can write the most general 
form of a CPTP map,

$$
\Lambda^{\prime}: \chi \mapsto \operatorname{tr}_{\mathrm{aux}} U\left(\chi \otimes \Pi_{|\mathrm{anc}\rangle}\right) U^{\dagger}
$$

From this we define

$$
\begin{aligned}
\Gamma_{\left.\Lambda^{\prime}, \Lambda ; \mid \text { anc }\right\rangle} & : \mathcal{B}\left(\mathcal{H}_{\text {in }}\right) \rightarrow \mathcal{B}\left(\mathcal{H}_{\text {in }} \otimes \mathcal{H}_{\text {aux }}\right) \\
& : \chi \mapsto U\left(\Lambda[\chi] \otimes \Pi_{\mid \text {anc }\rangle}\right) U^{\dagger} .
\end{aligned}
$$

The inverse map of $\Gamma_{\Lambda^{\prime}, \Lambda}$ obviously is $\left(\Gamma_{\Lambda^{\prime}, \Lambda}\right)^{\prime}=\operatorname{tr}_{\text {aux }}$ and by construction, $\operatorname{tr}\left(\Gamma_{\Lambda^{\prime}, \Lambda}\left[\rho_{\text {in }}\right]^{2}\right)=\operatorname{tr}\left(\Lambda\left[\rho_{\text {in }}\right]^{2}\right)$ holds for all $\rho_{\text {in }}$. Using the previous result, we find $\operatorname{tr}\left(\Gamma_{\Lambda^{\prime}, \Lambda}\left[\rho_{\text {in }}\right]^{2}\right)=$ $\operatorname{tr}\left(\Lambda\left[\rho_{\text {in }}\right]^{2}\right) \leq \operatorname{tr}\left(\rho_{\text {in }}^{2}\right)$, i.e., no state can become purer by the action of a reversible process.

Since a process that is reversible on all states cannot improve the purity of any state, one would guess that, on the other hand, a process which maps all states to a pure state cannot be reversible for any state. Such a process is called a purifying process, i.e., a CPTP map $\Lambda$ is purifying, if $\operatorname{tr}\left(\Lambda\left[\rho_{\text {in }}\right]^{2}\right)=1$ holds for any input state $\rho_{\text {in. }}$. The action of such a map, indeed, has to map any state to the same pure output state: Suppose $\Lambda\left[\rho_{1}\right] \neq \Lambda\left[\rho_{2}\right]$. Then for $\rho_{3}=\left(\rho_{1}+\rho_{2}\right) / 2$ we find $\Lambda\left[\rho_{3}\right]=\left(\Lambda\left[\rho_{1}\right]+\Lambda\left[\rho_{2}\right]\right) / 2$, which only can be pure, if $\Lambda\left[\rho_{1}\right]=\Lambda\left[\rho_{2}\right]$ in contradiction to our assumption. Thus a purifying process must destroy any information of the input state and cannot be reversible at all.

So, the properties of reversibility and output purity are completely incompatible for a physical process, if one demands these properties to hold on all possible input states. Our approach now is to require these properties only on a certain subset of states $\mathcal{M} \subset \mathcal{B}_{+}\left(\mathcal{H}_{\text {in }}\right)$. In Section 2 we analyze the properties of maps, which map at least two mixed states to pure states. The result of this investigation will completely characterize any such map. As an application of this result we will provide a lower bound on the trace distance of any two product states $\rho_{1} \otimes \sigma_{1}$ and $\rho_{2} \otimes \sigma_{2}$. In a brief excursion in Section 3 we will show that, if we allow probabilistic processes, non-trivial examples of reversible and purifying processes can easily be constructed. But we will also show that the definition of a probabilistic process to some extent contradicts the properties of a purifying process. In Section 4 we will then characterize any set of states, for which a deterministic reversible and purifying map exists and discuss in some detail the structure of such sets. Finally, we conclude in Section 5 .

\section{Purifying Processes of two states}

In the previous analysis we ruled out the possibility of a nontrivial process, which takes all states $\mathcal{B}_{+}\left(\mathcal{H}_{\text {in }}\right)$ to a corresponding pure state in $\mathcal{B}_{+}\left(\mathcal{H}_{\text {out }}\right)$. So the question arises, to what extent this also holds if one demands a pure output only for a subset of states $\mathcal{M} \subset \mathcal{B}_{+}\left(\mathcal{H}_{\text {in }}\right)$. More technically, for a CPTP map $\Lambda$, let us write $\operatorname{pur}(\Lambda)=\left\{\rho_{\text {in }} \in \mathcal{B}_{+}\left(\mathcal{H}_{\text {in }}\right)\right.$ $\left.\operatorname{tr}\left(\Lambda\left[\rho_{\text {in }}\right]^{2}\right)=1\right\}$ for the set of states which gets purified by the action of $\Lambda$. For a purifying process of $\mathcal{M}$ we demand $\mathcal{M} \subset \operatorname{pur}(\Lambda)$. In this section we will only deal with the most simple non-trivial case where only two states $\rho_{1}$ and $\rho_{2}$ are to be mapped onto a pure state, i.e., $\mathcal{M}=\left\{\rho_{1}, \rho_{2}\right\} \subset \operatorname{pur}(\Lambda)$.

Let us consider the case where we already have two purifying maps $\Lambda_{A}$ and $\Lambda_{B}$ acting on $\rho_{1}$ and $\rho_{2}$, and without loss of generality assume

$$
d\left(\Lambda_{A}\left[\rho_{1}\right], \Lambda_{A}\left[\rho_{2}\right]\right) \geq d\left(\Lambda_{B}\left[\rho_{1}\right], \Lambda_{B}\left[\rho_{2}\right]\right) .
$$

(Here, $d(\rho, \sigma)=\frac{1}{2} \operatorname{tr}|\rho-\sigma|$ with $|\chi|=\sqrt{\chi \chi^{\dagger}}$ denotes the trace distance of $\rho$ and $\sigma$.) Then there exists a CPTP map $\Omega$, such that up to a global unitary transformation, $\Lambda_{B}\left[\rho_{i}\right]=$ $\left(\Omega \circ \Lambda_{A}\right)\left[\rho_{i}\right]$ for $i=1,2$ : For two pure states $\Pi_{\left|\psi_{1}\right\rangle}$ and $\Pi_{\left|\psi_{2}\right\rangle}$ one can reduce the angle defined by $\sin \vartheta=d\left(\Pi_{\left|\psi_{1}\right\rangle}, \Pi_{\left|\psi_{2}\right\rangle}\right)$ to an arbitrary angle $\varphi<\vartheta$ via the CPTP map

$$
\Omega_{\varphi ;\left|\psi_{1}\right\rangle,\left|\psi_{2}\right\rangle}: \chi \mapsto \sum_{\alpha=1}^{3} A_{\alpha} \chi A_{\alpha}^{\dagger}
$$

with $A_{\alpha}$ being the Kraus operators [3]

$$
\begin{aligned}
& A_{1}=\left|\psi_{1}\right\rangle\left\langle\psi_{1}|+a| \psi_{1}^{\perp}\right\rangle\left\langle\psi_{1}^{\perp}\right|, \\
& A_{2}=\left(\sqrt{1-b^{2}}\left|\psi_{1}\right\rangle+\sqrt{b^{2}-a^{2}}\left|\psi_{1}^{\perp}\right\rangle\right)\left\langle\psi_{1}^{\perp}\right|, \\
& A_{3}=\mathbb{1}-\left|\psi_{1}\right\rangle\left\langle\psi_{1}|-| \psi_{1}^{\perp}\right\rangle\left\langle\psi_{1}^{\perp}\right|
\end{aligned}
$$

where $a=\tan \varphi \cot \vartheta, \quad b=\sin \varphi / \sin \vartheta, \quad$ and $\left|\psi_{1}^{\perp}\right\rangle \in$ $\operatorname{span}\left\{\left|\psi_{1}\right\rangle,\left|\psi_{2}\right\rangle\right\}$ is a normalized vector orthogonal to $\left|\psi_{1}\right\rangle$. Now let $\Pi_{\left|\psi_{i}\right\rangle}=\Lambda_{A}\left[\rho_{i}\right]$ and choose $\sin \varphi=$ $d\left(\Lambda_{B}\left[\rho_{1}\right], \Lambda_{B}\left[\rho_{2}\right]\right)$. Then, up to a global unitary transformation, $\Lambda_{B}\left[\rho_{i}\right]=\left(\Omega_{\varphi ;\left|\psi_{1}\right\rangle,\left|\psi_{2}\right\rangle} \circ \Lambda_{A}\right)\left[\rho_{i}\right]$ holds. Since we can mimic the action of $\Lambda_{B}$ on $\rho_{1}$ and $\rho_{2}$ by using the map $\Lambda_{A}$, we would always prefer $\Lambda_{A}$ in favor of $\Lambda_{B}$. Thus among all purifying processes of two states we are most interested in those which maximize the trace distance of the corresponding output states.

This trace distance of the output of a purifying map $\Lambda$ is upper bounded by a geometric quantity depending on $\rho_{1}$ and $\rho_{2}$, namely by the worst case distinguishability $\mathcal{D}\left(\rho_{1}, \rho_{2}\right)$ [4],

$$
d\left(\Lambda\left[\rho_{1}\right], \Lambda\left[\rho_{2}\right]\right) \leq \mathcal{D}\left(\rho_{1}, \rho_{2}\right)
$$

We now want to give a physical interpretation of this inequality: In quantum mechanics, an ensemble of pure states $\Pi_{\left|\phi_{j}\right\rangle}$ with probabilities $p_{j}>0$ (where $\sum_{j} p_{j}=1$ ) is described by the mixed state $\rho=\sum_{j} p_{j} \Pi_{\left|\phi_{j}\right\rangle}$. In general, many different ensembles lead to the same density operator $\rho$, and it is a prediction of quantum mechanics that it is impossible to physically distinguish between such different ensembles. Having said that, for a given mixed state $\rho$, a pure state $\Pi_{|\phi\rangle}$ may physically appear if and only if $\Pi_{|\phi\rangle}$ can be part of an ensemble that is represented by $\rho$, i.e., if and only if a positive number $p$ exists, such that $\rho-p \Pi_{|\phi\rangle}$ is positive semidefinite. Let us denote the collection of all such pure states $\Pi_{|\phi\rangle}$ by

$$
\begin{aligned}
\mathcal{Q}_{\rho} & =\left\{\Pi_{|\phi\rangle} \mid \exists p>0: \rho-p \Pi_{|\phi\rangle} \geq 0\right\} \\
& \left.\equiv\left\{\Pi_{|\phi\rangle}|| \phi\right\rangle \in \operatorname{supp} \rho\right\}
\end{aligned}
$$


where supp $\rho$ is the support of $\rho$, i.e., the orthocomplement of the kernel of $\rho$. The worst-case distinguishability is now defined as

$$
\begin{aligned}
\mathcal{D}\left(\rho_{1}, \rho_{2}\right) & =\inf _{\Pi_{\left|\phi_{i}\right\rangle} \in \mathcal{Q}_{\rho_{i}}} d\left(\Pi_{\left|\phi_{1}\right\rangle}, \Pi_{\left|\phi_{2}\right\rangle}\right) \\
& \equiv \min _{k}\left(\sin \vartheta_{k}\right),
\end{aligned}
$$

where $\vartheta_{k}$ denote the Jordan angles [5] between supp $\rho_{1}$ and $\operatorname{supp} \rho_{2}$.

Let us continue the physical motivation of Eq. (6). The maximal success probability for distinguishing two mixed states via a measurement ("minimum error discrimination") is given by [6,7]

$$
P_{\mathrm{MED}}\left(\rho_{1}, \rho_{2}\right)=\left(1+d\left(\rho_{1}, \rho_{2}\right)\right) / 2,
$$

where we assumed that both states have equal a priori probabilities. Hence $P_{\mathrm{MED}}$ is the average success probability for distinguishing the ensemble of pure states denoted by $\rho_{1}$ and $\rho_{2}$. In a physical experiment, each single measurement is performed on a pure state out of the ensembles, i.e., the task of the discrimination measurement is to distinguish between a state in $\mathcal{Q}_{\rho_{1}}$ and a state in $\mathcal{Q}_{\rho_{2}}$. The optimal success probability to distinguish between such two pure states in the worst case is given by

$$
\begin{aligned}
P_{\mathrm{WCD}} & =\inf _{\Pi_{\left|\phi_{i}\right\rangle} \in \mathcal{Q}_{\rho_{i}}} P_{\mathrm{MED}}\left(\Pi_{\left|\phi_{1}\right\rangle}, \Pi_{\left|\phi_{2}\right\rangle}\right) \\
& \equiv\left(1+\mathcal{D}\left(\rho_{1}, \rho_{2}\right)\right) / 2 .
\end{aligned}
$$

Since no deterministic process can increase the trace distance between two states [2], a purifying process of $\rho_{1}$ and $\rho_{2}$ must not deterministically increase the distance between any pair of pure states $\Pi_{\left|\phi_{1}\right\rangle} \in \mathcal{Q}_{\rho_{1}}$ and $\Pi_{\left|\phi_{2}\right\rangle} \in \mathcal{Q}_{\rho_{2}}$. This may serve as a physical motivation for the inequality in Eq. (6).

Can the bound in Eq. (6) always be achieved by some purifying process $\Lambda$ ? The answer is affirmative, but in order to verify this to a satisfactory level there is no way to avoid the awkwardness of an explicit construction of a map which reaches equality in Eq. (6):

Let us first briefly recall the concept of Jordan bases and Jordan angles (sometimes also called canonical bases and canonical angles) [5,8] of two subspaces $\mathcal{A}_{1} \subset \mathcal{H}$ and $\mathcal{A}_{2} \subset$ $\mathcal{H}$ : Orthonormal bases $\left|\psi_{1}^{k}\right\rangle$ of $\mathcal{A}_{1}$ and $\left|\psi_{2}^{k}\right\rangle$ of $\mathcal{A}_{2}$ are called Jordan bases, if

$$
\begin{aligned}
& \left\langle\psi_{1}^{k} \mid \psi_{2}^{l}\right\rangle=0 \\
& \text { for } k \neq l \text {, } \\
& \left\langle\psi_{1}^{k} \mid \psi_{2}^{k}\right\rangle=\cos \vartheta_{k} \\
& \text { for } k \leq \min _{i} \operatorname{dim} \mathcal{A}_{i} \text {. }
\end{aligned}
$$

Such bases always exist and $\vartheta_{k}$ are called the Jordan angles between $\mathcal{A}_{1}$ and $\mathcal{A}_{2}$.

The first step in the construction of the purifying map is to apply the distance-decreasing map $\Omega_{\varphi}$ defined in Eqns. (5a)(5d) on each pair of Jordan vectors $\left|\psi_{1}^{k}\right\rangle \in \operatorname{supp} \rho_{1}$ and $\left|\psi_{2}^{k}\right\rangle \in \operatorname{supp} \rho_{2}$, such that the distance is reduced to $\mathcal{D}\left(\rho_{1}, \rho_{2}\right)$ : We define the Kraus operators $A_{1}^{k}$ and $A_{2}^{k}$ for $k \leq \min _{i}$ rank $\rho_{i}$ analogously to Eqns. (5b) and (5c) and choose $\sin \varphi_{k}=\mathcal{D}\left(\rho_{1}, \rho_{2}\right)$. In order to complete the set of Kraus operators, we in addition define the projector $A_{3}=\mathbb{1}-\sum_{k} A_{1}^{k \dagger} A_{1}^{k}-\sum_{k} A_{2}^{k \dagger} A_{2}^{k}$ and write

$$
\begin{aligned}
& \tilde{\Omega}: \mathcal{B}\left(\mathcal{H}_{\text {in }}\right) \rightarrow \mathcal{B}\left(\mathcal{H}_{\text {in }}\right) \\
& \quad: \chi \mapsto \sum_{k} A_{1}^{k} \chi A_{1}^{k \dagger}+\sum_{k} A_{2}^{k} \chi A_{2}^{k \dagger}+A_{3} \chi A_{3}^{\dagger},
\end{aligned}
$$

Let $\Pi_{\left|\nu_{i}\right\rangle}$ be an arbitrary pure state in $\mathcal{Q}_{\rho_{i}}$. One finds that

$$
\begin{aligned}
\tilde{\Omega}\left[\Pi_{\left|\nu_{i}\right\rangle}\right]=\sum_{k} \operatorname{tr}\left(\Pi_{\left|\psi_{i}^{k}\right\rangle} \Pi_{\left|\nu_{i}\right\rangle}\right) \tilde{\Omega}\left[\Pi_{\left|\psi_{i}^{k}\right\rangle}\right] & \\
& +A_{3} \Pi_{\left|\nu_{i}\right\rangle} A_{3}^{\dagger} .
\end{aligned}
$$

By construction, $\Pi_{\left|\tilde{\psi}_{i}^{k}\right\rangle}=\tilde{\Omega}\left[\Pi_{\left|\psi_{i}^{k}\right\rangle}\right]$ is again pure with $\left\langle\tilde{\psi}_{i}^{k} \mid \tilde{\psi}_{j}^{l}\right\rangle=0$ for $k \neq l$ and $d\left(\Pi_{\left|\tilde{\psi}_{1}^{k}\right\rangle}, \Pi_{\left|\tilde{\psi}_{2}^{k}\right\rangle}\right)=\mathcal{D}\left(\rho_{1}, \rho_{2}\right)$. Furthermore $A_{3} \Pi_{\left|\nu_{i}\right\rangle} A_{3}^{\dagger} \neq 0$ only if $\operatorname{rank} \rho_{i}>\operatorname{rank} \rho_{j}$ for $j \neq i$.

Using the above properties of $\tilde{\Omega}$, it is straightforward to find a CPTP map $\tilde{E}: \mathcal{H}_{\text {in }} \rightarrow \mathcal{H}_{\text {in }} \otimes \mathcal{H}_{\text {aux }}$, such that the vectors $|k\rangle\left|\phi_{1}\right\rangle$ diagonalize $(\tilde{E} \circ \tilde{\Omega})\left[\rho_{1}\right]$ and the vectors $|k\rangle\left|\phi_{2}\right\rangle$ diagonalize $(\tilde{E} \circ \tilde{\Omega})\left[\rho_{2}\right]$, where $\langle k \mid l\rangle=\delta_{k l}$ and $d\left(\Pi_{\left|\phi_{1}\right\rangle}, \Pi_{\left|\phi_{2}\right\rangle}\right)=\mathcal{D}\left(\rho_{1}, \rho_{2}\right)$. Thus the map $\operatorname{tr}_{\text {in }} \circ \tilde{E} \circ \tilde{\Omega}$ is a map which reaches the bound in Eq. (6), i.e.,

$$
\mathcal{D}\left(\rho_{1}, \rho_{2}\right)=\max _{\Lambda} d\left(\Lambda\left[\rho_{1}\right], \Lambda\left[\rho_{2}\right]\right),
$$

where the maximum is taken over all CPTP maps $\Lambda$ satisfying $\left\{\rho_{1}, \rho_{2}\right\} \subset \operatorname{pur}(\Lambda)$. Furthermore, as already discussed in advance, due to Eq. (14), the maximizing map $\operatorname{tr}_{\text {in }} \circ \tilde{E} \circ \tilde{\Omega}$ together with the distance-decreasing map $\Omega_{\varphi}$ allows to mimic the action of any purifying map of the states $\rho_{1}$ and $\rho_{2}$.

This result characterizes the output of any process, which maps two input states to pure output states. For example one immediately finds that two states with overlapping support have a vanishing worst-case distinguishability and thus such states only can be mapped to identical pure states by a purifying process. In [4] the problem was investigated, how close the pure output states of a purifying map can get to a purification [9] 10] of the input states. The deviation from the optimal quality of such a purifying map was found to be limited by the difference $d\left(\rho_{1}, \rho_{2}\right)-\mathcal{D}\left(\rho_{1}, \rho_{2}\right)$. Furthermore the result in Eq. (14) turned out to be the key for the analysis of sets which can be mapped perfectly to their purifications [4].

In addition, the result in Eq. (14) can also be used as a general tool in quantum information theory, since results for pure states often are much simpler to obtain than results for mixed states. As an example, we provide a lower bound on the trace distance of any two product states $\rho_{1} \otimes \sigma_{1}$ and $\rho_{2} \otimes$ $\sigma_{2}$ :

$$
\begin{aligned}
& d\left(\rho_{1} \otimes \sigma_{1}, \rho_{2} \otimes \sigma_{2}\right)^{2} \geq \\
& 1-\left(1-\mathcal{D}\left(\rho_{1}, \rho_{2}\right)^{2}\right)\left(1-d\left(\sigma_{1}, \sigma_{2}\right)^{2}\right) .
\end{aligned}
$$

(From this inequality in particular $d\left(\rho_{1}, \rho_{2}\right) \geq \mathcal{D}\left(\rho_{1}, \rho_{2}\right)$ follows by setting $\sigma_{1}=\sigma_{2}$.) This inequality follows by applying 
a map for which

$$
\begin{aligned}
& \rho_{1} \otimes \sigma_{1} \mapsto \Pi_{\left|\phi_{1}\right\rangle} \otimes\left(q_{1} \Pi_{|0\rangle}+\left(1-q_{1}\right) \Pi_{|1\rangle}\right), \\
& \rho_{2} \otimes \sigma_{2} \mapsto \Pi_{\left|\phi_{2}\right\rangle} \otimes\left(\left(1-q_{2}\right) \Pi_{|0\rangle}+q_{2} \Pi_{|1\rangle}\right) .
\end{aligned}
$$

Such a mapping can be implemented by a CPTP map for appropriate $q_{1}, q_{2}$ satisfying $q_{1}+q_{2}=1+d\left(\sigma_{1}, \sigma_{2}\right)$ and $\Pi_{\left|\phi_{i}\right\rangle}$ satisfying $d\left(\Pi_{\left|\phi_{1}\right\rangle}, \Pi_{\left|\phi_{2}\right\rangle}\right)=\mathcal{D}\left(\rho_{1}, \rho_{2}\right)$, since then for the first system one applies the purifying map $\operatorname{tr}_{\text {in }} \circ \tilde{E} \circ \tilde{\Omega}$ and for the second system one applies a minimum error discrimination of $\sigma_{1}$ and $\sigma_{2}$. Now using the fact that a CPTP map cannot increase the trace distance, it is straightforward to obtain Eq. (15).

\section{Probabilistic Purifying Processes}

Although we want to concentrate on deterministic processes, in this section we wish to briefly discuss the properties of probabilistic purifying processes. We exclude probabilistic processes $\bar{\Lambda}$ from our considerations, for which $\operatorname{tr} \bar{\Lambda}[\rho]=0$ for some $\rho \in \mathcal{M}$, i.e., we call a process probabilistic on $\mathcal{M}$, only if for any state in $\mathcal{M}$ the success probability of the process is non-zero.

A simple example of a probabilistic purifying process is a process, which first performs an unambiguous state discrimination [11,8] between the possible input states and then uses the unambiguous information to create a purification of the input state: In the language of probabilistic processes, unambiguous state discrimination of a set of states $\rho_{i}$ is a probabilistic map which maps $\rho_{i}$ to $p_{i} \Pi_{|i\rangle}$, where $p_{i}$ is the success probability of unambiguously identifying $\rho_{i}$ and $\langle i \mid j\rangle=\delta_{i j}$. In Ref. [12] it was shown, that a probabilistic unambiguous state discrimination process for a set $\mathcal{M}$ exists, if and only if

$$
\operatorname{supp} \rho_{i} \nsubseteq \sum_{j \neq i} \operatorname{supp} \rho_{j}, \quad \forall \rho_{i} \in \mathcal{M}
$$

Hence, if one applies unambiguous state discrimination on such a set $\mathcal{M}$, in case of a successful discrimination one can map each state $\Pi_{|i\rangle}$ to a purification $\Pi_{\left|\psi_{i}\right\rangle}$ of $\rho_{i}$. This map is purifying as well as reversible on $\mathcal{M}$ (with the reversible map being the partial trace over the purifying system) and it is successful, whenever the unambiguous state discrimination process succeeds.

However, there is a good reason not to deepen the analysis of probabilistic processes as a proper variant of purifying processes: Physically, the information of a successful application of a probabilistic map is provided as a bit of classical information. Thus for a probabilistic purifying map $\bar{\Lambda}$ one can equivalently write the deterministic map

$$
\Lambda: \chi \mapsto \bar{\Lambda}[\chi]+(\operatorname{tr} \chi-\operatorname{tr} \bar{\Lambda}[\chi]) \Pi_{|?\rangle},
$$

where $\Pi_{|?\rangle}$ is a state that is orthogonal to all output operators $\bar{\Lambda}[\chi]$. However, the output of $\Lambda$ is not pure, unless $\bar{\Lambda}$ is already deterministic and purifying.

\section{Purifying and Reversible Processes}

We now want to combine the purifying property of a deterministic process with the feature of reversibility. Since we already noticed that processes which are reversible on all states cannot increase the purity of any state (although it possible to decrease the purity, e.g. using the map $E_{\sigma}$ defined in Eq. (11), in the fashion of Section 2 we demand reversibility only on a subset of states $\mathcal{M}$. We call a CPTP map $\Lambda$ reversible on $\mathcal{M}$ if one can find a CPTP map $\Lambda^{\prime}$, such that $\left(\Lambda^{\prime} \circ \Lambda\right)\left[\rho_{\text {in }}\right]=\rho_{\text {in }}$ for all $\rho_{\text {in }} \in \mathcal{M}$. Let us, again, formalize this property: For a CPTP map $\Xi: \mathcal{B}\left(\mathcal{H}_{\text {in }}\right) \rightarrow \mathcal{B}\left(\mathcal{H}_{\text {in }}\right)$, let $\operatorname{ID}(\Xi)=\left\{\rho_{\text {in }} \in \mathcal{B}_{+}\left(\mathcal{H}_{\text {in }}\right) \mid \Xi\left[\rho_{\text {in }}\right]=\rho_{\text {in }}\right\}$ be the set of states that are unchanged by the action of $\Xi$. Thus for a reversible map $\Lambda$ on $\mathcal{M}$, we demand that one can find a CPTP map $\Lambda^{\prime}$, such that $\mathcal{M} \subset \operatorname{ID}\left(\Lambda^{\prime} \circ \Lambda\right)$. Note, that $\Lambda^{\prime}$ does not need to be unique. Now a map $\Lambda$ is purifying and reversible on $\mathcal{M}$, if and only if one can find a map $\Lambda^{\prime}$, such that $\mathcal{M} \subset \operatorname{pur}(\Lambda) \cap \operatorname{ID}\left(\Lambda^{\prime} \circ \Lambda\right)$. It is possible to completely characterize any such set $\mathcal{M}$ :

Theorem 1 A reversible and purifying process for a set of states $\mathcal{M} \subset \mathcal{B}_{+}\left(\mathcal{H}_{\mathrm{in}}\right)$ exists, if and only if for appropriate vector spaces $\mathcal{H}_{C}^{i}, \mathcal{H}_{A}^{i}$ and $\mathcal{H}_{B}^{i}$, satisfying $\mathcal{H}_{\mathrm{in}} \otimes \mathcal{H}_{C}^{i} \cong$ $\mathcal{H}_{A}^{i} \otimes \mathcal{H}_{B}^{i}$, one can find mixed states $\sigma_{B}^{i} \in \mathcal{B}_{+}\left(\mathcal{H}_{B}^{i}\right)$ and $\omega_{C}^{i} \in \mathcal{B}_{+}\left(\mathcal{H}_{C}^{i}\right)$, and unitary transformations $U_{i}$, such that $\mathcal{M}=\bigcup_{i} \mathcal{M}_{i}$ with $\mathcal{M}_{i} \perp \mathcal{M}_{j}, i \neq j$ and

$$
\begin{aligned}
& \left\{\rho \otimes \omega_{C}^{i} \mid \rho \in \mathcal{M}_{i}\right\} \subset \\
& \left.\quad\left\{U_{i}\left(\Pi_{|\phi\rangle} \otimes \sigma_{B}^{i}\right) U_{i}^{\dagger}|| \phi\right\rangle \in \mathcal{H}_{A}^{i}\right\} .
\end{aligned}
$$

In Theorem $1 \mathcal{M}_{i} \perp \mathcal{M}_{j}$ if $\operatorname{tr}(\rho \sigma)=0$ for all $\rho \in \mathcal{M}_{i}$ and $\sigma \in \mathcal{M}_{j}$. Sets $\mathcal{M}_{i}$, which satisfy Eq. (20) are called essentially pure, i.e., a reversible and purifying process for $\mathcal{M}$ exists, if and only if $\mathcal{M}$ is an orthogonal union of essentially pure sets. Furthermore note, that basically, essentially pure sets are such sets which are generated by applying the map $E_{U} \circ E_{\sigma}$ on a set of pure states.

Proof (Theorem 11) In [4] it was shown, that $\mathcal{M}$ is an orthogonal union of essentially pure sets, if and only if a perfect purifier of $\mathcal{M}$ exists. A perfect purifier is a CPTP map, which maps any state in $\mathcal{M}$ to one of its purifications in $\mathcal{B}_{+}\left(\mathcal{H}_{\text {in }} \otimes \mathcal{H}_{\text {aux }}\right)$. Hence a perfect purifier $\Lambda$ of $\mathcal{M}$ in particular satisfies $\mathcal{M} \subset \operatorname{pur}(\Lambda)$ and $\mathcal{M} \subset \operatorname{ID}\left(\operatorname{tr}_{\text {aux }} \circ \Lambda\right)$, i.e., it is purifying and reversible.

For the converse assume that a reversible and purifying map $\Lambda$ for $\mathcal{M}$ exists. Then $\mathcal{M} \subset \operatorname{pur}(\Lambda)$ and one can find a CPTP map $\Lambda^{\prime}$, such that $\mathcal{M} \subset \operatorname{ID}\left(\Lambda^{\prime} \circ \Lambda\right)$. The map $\Gamma_{\Lambda^{\prime}, \Lambda}$ defined in Eq. (3) thus satisfies $\operatorname{pur}\left(\Gamma_{\Lambda^{\prime}, \Lambda}\right)=\operatorname{pur}(\Lambda) \supset \mathcal{M}$ and $\operatorname{ID}\left(\operatorname{tr}_{\text {aux }} \circ \Gamma_{\Lambda^{\prime}, \Lambda}\right)=\operatorname{ID}\left(\Lambda^{\prime} \circ \Lambda\right) \supset \mathcal{M}$ and hence $\Gamma_{\Lambda^{\prime}, \Lambda}$ is a perfect purifier of $\mathcal{M}$. Using again the result in [4], it follows that $\mathcal{M}$ is an orthogonal union of essentially pure sets.

Although Theorem 1 completely characterizes all sets for which a reversible and purifying process exists, it is in general not straightforward to test whether a set is of the structure 
as specified in Eq. (20). Only for the case where $\mathcal{M}$ consists of only two states, an operational necessary and sufficient criterion is known [4]: The set $\mathcal{M}=\left\{\rho_{1}, \rho_{2}\right\}$ is essentially pure or $\rho_{1} \perp \rho_{2}$ if and only if $\mathcal{D}\left(\rho_{1}, \rho_{2}\right)=d\left(\rho_{1}, \rho_{2}\right)$. In the general case only some necessary operational conditions can be derived. The most obvious necessary criterion is, that in an essentially pure set all states must share the same spectrum. Another example of a necessary criterion is, that the Jordan angles between the support of any two states taken from an essentially pure set have to be completely degenerate. But these two properties are not sufficient for an essentially pure set, as the following simple counter-example demonstrates:

$$
\begin{aligned}
& \rho_{1}=p \Pi_{|0\rangle}+(1-p) \Pi_{|1\rangle} \\
& \rho_{2}=p \Pi_{\left|\nu^{+}\right\rangle}+(1-p) \Pi_{\left|\nu^{-}\right\rangle},
\end{aligned}
$$

where $\left|\nu^{ \pm}\right\rangle=\frac{1}{2}( \pm|0\rangle+|1\rangle \pm|2\rangle+|3\rangle)$ and $0<p<\frac{1}{2}$.

As a final remark let us note that it is possible to simplify the definition of essentially pure sets: A set of states $\mathcal{M} \subset \mathcal{B}_{+}\left(\mathcal{H}_{\text {in }}\right)$ with $\rho_{0} \in \mathcal{M}$ is essentially pure if and only if one can find a unitary transformation $U$ on $\mathcal{H}_{\text {in }} \otimes \mathcal{H}_{\text {aux }}$ and normalized vectors $|\rho\rangle \in \mathcal{H}_{\text {aux }}$ corresponding to each $\rho \in \mathcal{M}$, such that for each $\rho \in \mathcal{M}$,

$$
\rho \otimes \Pi_{\left|\rho_{0}\right\rangle}=U\left(\rho_{0} \otimes \Pi_{|\rho\rangle}\right) U^{\dagger}
$$

holds. From the proof of Theorem 1 in [4] it is clear that in Eq. (20) one always can choose $\omega_{C}$ to be pure. Now the dimension of the kernel of each element on the left hand side of Eq. (20) is given by $\operatorname{dim}\left(\mathcal{H}_{\text {in }}\right) \operatorname{dim}\left(\mathcal{H}_{C}\right)-\operatorname{rank} \rho$ while on the right hand side we find $\operatorname{dim}\left(\mathcal{H}_{A}\right) \operatorname{dim}\left(\mathcal{H}_{B}\right)-\operatorname{rank} \rho$. One readily extends $\mathcal{H}_{C}$ and $\mathcal{H}_{B}$ such that $\operatorname{dim} \mathcal{H}_{B}$ is an integer multiple of $\operatorname{dim}\left(\mathcal{H}_{\text {in }}\right)$. Then after a suitable rotation $U^{\prime}$ on $\mathcal{H}_{A} \otimes \mathcal{H}_{B}$, one has $U^{\prime}\left(\Pi_{|\phi\rangle} \otimes \sigma_{B}\right) U^{\prime \dagger}=\left(\rho_{0} \otimes \Pi_{|0\rangle}\right) \otimes$ $\Pi_{|\phi\rangle}$. Identifying $\Pi_{|0\rangle} \otimes \Pi_{|\phi\rangle}$ with $\Pi_{|\rho\rangle}$ finishes the proof of Eq. (22).

\section{Conclusions}

In summary we have analyzed deterministic physical processes which are reversible or purifying, with particular focus on the combination of both properties. First we have shown that the properties of reversibility and purity of a physical processes are completely incompatible, as long as reversibility or purity is required to hold for any input state. For certain restricted sets, however, one can combine these properties. We investigated the case, where only two input states are mapped to pure states. It turned out that the trace distance of the output states of such a map is limited by the worstcase distinguishability of the input states. A map was provided, which always reaches this bound. Some applications of this result in quantum information theory were presented. For probabilistic processes we used unambiguous state discrimination to build a non-trivial example of a purifying and reversible process. We finally characterized all sets of states, for which a deterministic purifying and reversible process exists and it turned out that such sets have to be pure up to a common mixed contribution. Despite this result and the existence of an operational criterion for such essentially pure sets in the case, where the set consists of only two states, no operational necessary and sufficient condition for larger essentially pure sets was provided. Such criteria will be subject to further research. Furthermore, although some properties of reversible processes where presented here, another direction of future work will be to deepen the understanding of such processes.

Acknowledgements This work was partially supported by the European Commission (Integrated Projects SECOQC and SCALA).

\section{References}

1. W. Forrest Stinespring. Positive functions on $C^{*}$-algebras. Proc. Amer. Math. Soc., 6:211-216, 1955.

2. Michael A. Nielsen and Isaac L. Chuang. Quantum Computation and Quantum Information. Cambridge University Press, Cambridge, 2000.

3. K. Kraus. States, Effects and Operations. Springer-Verlag Berlin, 1983.

4. Matthias Kleinmann, Hermann Kampermann, Tim Meyer, and Dagmar Bruß. Physical purification of quantum states. Phys. Rev. A, 73:062309, 2006.

5. Gilbert W. Stewart and Ji-guang Sun. Matrix Pertubation Theory. Academic Press, San Diego, 1990.

6. Carl W. Helstrøm. Quantum Detection and Estimation Theory. Academic, New York, 1976.

7. Ulrike Herzog and János A. Bergou. Distinguishing mixed quantum states: Minimum-error discrimination versus optimum unambiguous discrimination. Phys. Rev. A, 70:022302, 2004.

8. Terry Rudolph, Robert W. Spekkens, and Peter S. Turner. Unambiguous discrimination of mixed states. Phys. Rev. A, 68:010301(R), 2003.

9. Lane P. Hughston, Richard Jozsa, and William K. Wootters. A complete classification of quantum ensembles having a given density matrix. Phys. Lett. A, 183:14-18, 1993.

10. Angelo Bassi and GianCarlo Ghirardi. A general scheme for ensemble purification. Phys. Lett. A, 309:24-28, 2003.

11. Gregg Jaeger and Abner Shimony. Optimal distinction between two non-orthogonal quantum states. Phys. Lett. A, 197:83-87, 1995.

12. Yuan Feng, Runyao Duan, and Ying Mingsheng. Unambiguous discrimination between mixed quantum states. Phys. Rev. A, 70:012308, 2004. 